\section{6d EFFECTS OF OLFACTORY STIMULUS BY ODOUR ON CEREBRAL BLOOD FLOW AND PERIPHERAL BLOOD OXYGEN LEVELS IN MULTIPLE CHEMICAL SENSITIVITY}

${ }^{1} \mathrm{~K}$ Azuma*, ${ }^{2} \mathrm{U}$ Uchiyama, ${ }^{2} \mathrm{M}$ Tanigawa, ${ }^{3} \mathrm{I}$ Bamba, ${ }^{4} \mathrm{M}$ Azuma, ${ }^{5} \mathrm{H}$ Takano, ${ }^{2} \mathrm{~T}$ Yoshikawa, ${ }^{6} \mathrm{~K}$ Sakabe. ${ }^{1}$ Kindai University Faculty of Medicine, Osakasayama, Japan; ${ }^{2}$ Louis Pasteur Centre for Medical Research, Kyoto, Japan; ${ }^{3}$ Tokyo Gakugei University, Koganei, Japan; ${ }^{4}$ Kio University Faculty of Health Sciences, Kitakatsuragi-gun, Japan; ${ }^{5}$ Kyoto University, Kyoto, Japan; ${ }^{6}$ Tokai University School of Medicine, Isehara, Japan

\subsection{6/oemed-2018-ICOHabstracts.815}

Introduction Multiple chemical sensitivity (MCS), often called idiopathic environmental intolerance, is a chronic acquired disorder characterised by nonspecific symptoms attributed to exposure to common odorous chemicals. We previously reported significant activations in the prefrontal cortex (PFC) during olfactory stimulation using several different odorants in patients with MCS compared with controls. Previous clinical observations demonstrated differences in oxygen partial pressure in peripheral venous blood between patients with MCS and controls. Our objective is to investigate peripheral blood oxygen levels during olfactory stimulation to characterise patients with MCS.

Methods We investigated changes in the prefrontal area using near-infrared spectroscopic (NIRS) imaging and those in peripheral arterial blood oxygen saturation $\left(\mathrm{SpO}_{2}\right)$ using a pulse oximeter during olfactory stimulation with odorants $(\gamma$ undecalactone) at three concentrations (zero, odour recognition threshold, and normal perceived odour level) in 11 patients with MCS and 10 controls. We also examined their oxygen partial pressures in peripheral venous blood at normal condition before the test.

Results We observed significant activations in the PFC during olfactory stimulation in patients with MCS. The $\mathrm{SpO}_{2}$ in peripheral arterial blood in patients with MCS remained higher than that in controls during olfactory stimulation tests. The $\mathrm{SpO}_{2}$ remarkably decreased in patients with MCS compared with that in controls during olfactory stimulus at normal perceived odour level and the recovery of the $\mathrm{SpO}_{2}$ after the stimulus was delayed in these patients. No significant differences in oxygen partial pressures of peripheral venous blood were observed.

Conclusion Patients with MCS exhibited stronger brain responses to odours at normally perceived levels. Changes in $\mathrm{SpO}_{2}$ in the peripheral arterial blood suggest that MCS might result in poor oxygen supply to the peripheral tissues or inadequately control the oxygen supply.

\section{6 e MOBILE PHONE USE AND ONSET OF SYMPTOMS. AN UPDATE OF THE EVIDENCE FROM PROSPECTIVE COHORT STUDIES}

L Hillert. Institute of Environmental Medicine, Karolinska Institutet, Stockholm, Sweden

\subsection{6/oemed-2018-ICOHabstracts.816}

Parallel to the rapid increase in mobile phone use worldwide concerns have been raised about various health effects. Acute symptoms have by some individuals been reported to be triggered when holding the phone to the head during calls or being close to a base station for mobile telephony. Symptoms vary, but headache, fatigue and difficulties concentrating are among the most commonly reported. Exposure to radiofrequency fields have been proposed as the cause of the symptoms. Double blind provocation studies have however failed to provide support for such a causal link. In spite of this, some people are still convinced that their ill health is caused by exposure to radiofrequency fields from mobile phones. It has also been suggested that a greater number of people than those who report experiencing these reactions to radiofrequency fields might be affected without being aware of the triggering factor. In theory, long term effects after repeated frequent exposure to radiofrequency fields from mobile phones may exist regardless of whether acute effects exists or not. The first studies on a possible relation between mobile phone use and symptoms in the general population were cross-sectional studies. This study design gives little information on possible causal relationship and suffers from a risk of reverse causality as well as a nocebo effect. Lately however, a number of prospective cohort studies have been published. This presentation will provide an update of the knowledge on mobile phone use and the onset of symptoms from these studies.

\section{$1716 f$ ENVIRONMENTAL INTOLERANCE - A CLINICAL PERSPECTIVE}

$1,2 \mathrm{M}$ Tondel. 'Occupational and Environmental Medicine, Uppsala University Hospital, Uppsala, Sweden; ${ }^{2}$ Occupational and Environmental Medicine, Department of Medical Sciences, Uppsala University, Uppsala, Sweden

\subsection{6/oemed-2018-ICOHabstracts.817}

Environmental intolerance is relatively prevalent in the general population, recognised by the patients as highly variable symptoms attributed to different perceived harmful exposures such as indoor environment, chemicals, odours, electromagnetic fields and noise. It is not uncommon with progression from a few triggers and symptoms to a multifaceted complex of symptoms triggered by multiple factors. Typically, the symptoms occur at very low exposure levels, below thresholds of acute toxicologic effects and at levels tolerated by a large majority of the population. To understand the background of environmental intolerance, a multi-professional approach is needed with knowledge of allergy, public health, toxicology, physics, chemistry, psychiatry and psychology. In Uppsala we have developed a model where the patients have individual consultations with a medical doctor, an industrial hygienist and a psychologist, respectively. The overall aim is a thorough evaluation of symptoms, exposure and temporal relationship to rule out a medical disease aiming at curative treatment. A well-defined disease is only established in a minority of the cases. Instead, a holistic approach is needed including analysing a range of aspects that may contribute to the condition. The attitude towards the patient, in the professional team, is characterised by active listening, seriousness, confidence building and sufficient time for the patient to describe symptoms and triggers. The final assessment of the patient is made by the physician after discussing the results of the examinations made by other members of the team. By a respectful response aiming for a trustful relationship a discussion can then continue with the patient giving objective information, based on scientific state of the art, related to environmental intolerance. An individual treatment plan includes, if necessary, medical treatment and cognitive behavioural therapy to encourage the 
patient to refrain from an avoidance behaviour for the triggers, thus improving quality of life and social functioning.

\section{ORGANOPHOSPHORUS PESTICIDE-INDUCED NEUROTOXICITY IN HUMAN APPLICATORS AND ANIMALS WITH COMPARABLE EXPOSURES}

W Kent Anger. Oregon Health and Science University, Portland, OR, USA

10.1136/oemed-2018-ICOHabstracts.818

Aim of special session Preventable chlorpyrifos exposures produce durable behavioural deficits in humans and animals; animal studies suggest oxidative stress contributes to cognitive deficits.

James R. Olson ${ }^{1}$, Diane S. Rohlman ${ }^{2}$, W. Kent Anger ${ }^{3}$, Pamela J. Lein ${ }^{4}$, Fayssal M. Farahat ${ }^{5}$

${ }^{1}$ University at Buffalo, Buffalo, NY, USA

${ }^{2}$ University of Iowa, Iowa City, IA, USA

${ }^{3}$ Oregon Health \& Science University, Portland, OR, USA

${ }^{4}$ University of California Davis, Davis, CA, USA

${ }^{5}$ Menoufia University, Shebin El-Kom, Egypt

\section{9a BIOMARKERS OF CHLORPYRIFOS AND PROFENOFOS EXPOSURE AND EFFECT IN ADULT AND ADOLESCENT EGYPTIAN COTTON FIELD WORKERS}

JR Olson. University at Buffalo, Buffalo, NY, USA

10.1136/oemed-2018-ICOHabstracts.819

Introduction Chlorpyrifos (CPF) and profenofos (PFF) are organophosphorus (OP) pesticides that are applied seasonally in Egypt to cotton fields. Urine trichloro-2-pyridinol (TCPy), a specific CPF metabolite, and 4-bromo-2-chlorophenol (BCP), a specific PFF metabolite, are biomarkers of exposure, while inhibition of blood butyrylcholinesterase (BChE) and acetylcholinesterase (AChE) activities are effect biomarkers which may be associated with neurotoxicity.

Methods Urine TCPy and BCP and blood BChE and AChE activities were measured in 37 adult Egyptian Ministry of Agriculture workers during and after 9-17 consecutive days of CPF application followed by PFF (8-10 days), and a second CPF application (5 days) in 2008. In a separate longitudinal study, 57 adolescent applicators and 38 age-matched nonapplicators were studied over 10 months in 2010 .

Results During the OP applications, mean TCPy and BCP levels were significantly higher than baseline levels and remained elevated following the application periods. Peak urinary BCP and peak TCPy levels for individuals (ranging from 13.4 to 8052 and 16.4 to $30,107 \mathrm{ug} / \mathrm{g}$ creatinine, respectively) were also highly correlated $(\mathrm{r}=0.77, \mathrm{p}<0.001)$. In adults, a significant inverse correlation was observed between urinary TCPy and blood BChE and AChE activities. In the adolescent study, the mean peak TCPy levels were less than the adults, but the exposure-effect relationship for BuChE inhibition was similar to adults. Both adolescent groups had elevated TCPy and depressed BChE which persisted for 4-7 weeks after spraying ended.

Conclusion Biomarker data in the adolescent non-applicators, which mirrored that of the applicators, indicated that the non-applicators received environmental CPF exposures. The variability in environmental and occupational exposures suggest that job title and work location should not be used as the sole basis for categorising OP exposures. Together, these results can serve to guide future investigations in assessing health risks of OPs and guide efforts to reduce exposures.

\section{$1639 \mathrm{~b}$ OCCUPATIONAL PESTICIDE EXPOSURE AMONG EGYPTIAN ADOLESCENTS: CHANGES OVER TIME}

DS Rohlman. University of lowa, lowa City, lowa, USA

\subsection{6/oemed-2018-ICOHabstracts.820}

Introduction While the impact of acute high exposures (i.e., poisoning) to organophosphorus insecticides is well understood, the impact of low level exposures, particularly on neurobehavioral functioning, is still under debate. Even less information is available regarding cumulative exposure, particularly among adolescents who may be working in agriculture. The goal was to examine the impact of chlorpyrifos exposure on biomarkers of exposure and neurobehavioral performance in adolescents across an application season.

Methods Male Egyptian adolescents (applicators and non-applicators) were assessed 35 times before, during and after the pesticide application season. At each session, participants $(n=89)$ completed a neurobehavioral test battery and urine was collected for analysis of the chlorpyrifos metabolite 3,5,6trichloro-2 pyridinol (TCPy) (biomarker of exposure). Cumulative urinary TCPy over the study period was used to classify participants into low $(<$ median $)$ and high $(\geq$ median $)$ exposure groups.

Results Urinary TCPy increased during application with recovery following the end of application. High exposed participants had significantly elevated metabolite levels throughout the 10 month study period. Deficits in motor skills and slower reaction times, along with deficits in executive function and short-term memory were found between the high and low exposure groups. Changes in neurobehavioral performance across the application season indicate a pattern of impaired performance among the high exposed compared to the low exposed. Deficits increased during the application season and remained for months after application ended.

Conclusion The findings indicate that neurobehavioral deficits increase during the application season, as exposure also increases, and remain after the application ends, even when the biomarkers of exposure are reduced. This is particularly important when considering the developmental changes that occur during adolescence. An intervention to reduce pesticide exposure has been implemented in this population.

(Supported by NIEHS and the Fogarty Institute R21 ES017223 and R01 ES022163, Rohlman).

\section{C ORGANOPHOSPHORUS PESTICIDE NEUROTOXICITY IN EGYPTIAN APPLICATORS}

WK Anger. Oregon Health and Science University, Portland, OR, USA

\subsection{6/oemed-2018-ICOHabstracts.821}

Introduction Chronic occupational exposure to organophosphorus pesticides (OPs) is consistently associated with deficits on neurobehavioral tests when compared to unexposed groups. However, a dose-response relationship has not been established, leading some to doubt an association between 